UDK 528.8

\title{
USING PIXEL-BASED AND OBJECT-BASED METHODS TO CLASSIFY URBAN HYPERSPECTRAL FEATURES
}

\author{
Ahmad HADAVAND ${ }^{1}$, Mehdi MOKHTARZADEH ${ }^{2}$, Mohammad Javad Valadan ZOEJ ${ }^{2}$, \\ Saeid HOMAYOUNI ${ }^{3}$, Mohammad SAADATSERESHT ${ }^{1}$
}

\author{
${ }^{1}$ Department of Surveying and Geomatics Engineering, College of Engineering, \\ University of Tehran, Tehran, Iran \\ ${ }^{2}$ Faculty of Geodesy and Geomatics, K. N. Toosi University, Tehran, Iran \\ ${ }^{3}$ Department of Geography, University of Ottawa, Ottawa, Canada \\ E-mail: ahadavand@ut.ac.ir (corresponding author)
}

Received 13 April 2015; accepted 12 September 2016

\begin{abstract}
Object-based image analysis methods have been developed recently. They have since become a very active research topic in the remote sensing community. This is mainly because the researchers have begun to study the spatial structures within the data. In contrast, pixel-based methods only use the spectral content of data. To evaluate the applicability of object-based image analysis methods for land-cover information extraction from hyperspectral data, a comprehensive comparative analysis was performed. In this study, six supervised classification methods were selected from pixel-based category, including the maximum likelihood (ML), fisher linear likelihood (FLL), support vector machine (SVM), binary encoding (BE), spectral angle mapper (SAM) and spectral information divergence (SID). The classifiers were conducted on several features extracted from original spectral bands in order to avoid the problem of the Hughes phenomenon, and obtain a sufficient number of training samples. Three supervised and four unsupervised feature extraction methods were used. Pixel based classification was conducted in the first step of the proposed algorithm. The effective feature number (EFN) was then obtained. Image objects were thereafter created using the fractal net evolution approach (FNEA), the segmentation method implemented in eCognition software. Several experiments have been carried out to find the best segmentation parameters. The classification accuracy of these objects was compared with the accuracy of the pixel-based methods. In these experiments, the Pavia University Campus hyperspectral dataset was used. This dataset was collected by the ROSIS sensor over an urban area in Italy. The results reveal that when using any combination of feature extraction and classification methods, the performance of object-based methods was better than pixel-based ones. Furthermore the statistical analysis of results shows that on average, there is almost an 8 percent improvement in classification accuracy when we use the object-based methods.
\end{abstract}

Keywords: hyperspectral, pixel-based, object-based, feature extraction, segmentation.

\section{Introduction}

Rich spectral content of hyperspectral data has brought about the new possibilities as well as new challenges for image analysis and information extraction. Hyperspectral data can be used to detect and identify different terrestrial features such as minerals (Kruse et al. 2003), urban features (Roessner et al. 2001), and land-covers (Rogan, Chen 2004). In addition to providing spectral information, hyperspectral imagery sometimes provides a high spatial resolution (e.g., with respect to airborne data). Both spectral and spatial information are important for detecting, classifying, and identifying the features of various phenomena (Roessner et al. 2001; Dell'Acqua et al. 2004). However, new processing tools and methods are needed to obtain reliable and accurate geospatial information.

Due to variations in spatial resolution, information extraction from hyperspectral data involves several strategies. In the case of classic pixel-based classification, there are two main categories of approaches: whole-pixel and sub-pixel approaches. Blaschke (2010) introduced three cases concerning the relative size of objects and spatial resolution. He represent the transition from sub-pixel methods to pixel-based and 
object-based methods by increasing the spatial resolution of the sensors (Blaschke 2010).

The primary methods used for the classification of remote sensing data were based on pattern recognition algorithms. These algorithms were originally developed by experts and incorporate statistical bases, such as both the Bayes (Bischof et al. 1992) and Maximum Likelihood (Paola, Schowengerdt 1995) algorithms. Moreover, these algorithms are used for pixel-based classification. Remote sensing experts tried to develop dedicated methods such as SAM (Kruse et al. 1993), or non-statistic methods such as SVM (Melgani, Bruzzone 2004) and Synergethics (Müller et al. 2013) based theories in pixel-based classification.

Acquiring high resolution imagery in urban areas causes to obtain a sufficient number of neighboring pixels belonging to same land-cover class. High resolution imagery creates a spatial correlation between pixels. Consequently, classification methods need to be capable of assessing both spectral and spatial information in the image. In addition to the classic methods (Kettig, Landgrebe 1976), new classification methods have recently been proposed for this purpose (Dell'Acqua et al. 2004; Plaza 2008; Tarabalka et al. 2009, 2010; Salehi et al. 2012). Two main challenges that arise when using these methods are as follows (Tarabalka 2010):

- Extracting the spatial features from an image.

- Combining the spectral and spatial information within the classification process.

Different methods have been developed with the aim of addressing these problems. Among these methods, the Extraction and Classification of Homogeneous Objects (ECHO) (Kettig, Landgrebe 1976) was the focus of one of the first research studies. Based on this study, various methods have been developed and proposed. The central idea behind these methods is that neighboring pixels probably belong to the same landcover class (Jackson, Landgrebe 2002; Dell'Acqua et al. 2004). Most common concepts that may help achieve this goal are as follows: the morphological profile and derivative morphological profile (Pesaresi, Benediktsson 2001; Palmason et al. 2003), morphological operators (Soille 2003), the extended morphological profile (Benediktsson et al. 2005) composite kernels (CampsValls et al. 2006), and image texture (Tsai et al. 2006).

The common point of pixel-based methods is the way they extract and use the spatial information of an image scene. These methods add the spatial information extracted from a predefined neighboring window to the feature vector of the central pixel. Despite the computational complexity of these methods, they cannot take into account every spatial structure, because of simplifications they use in defining spatial structures. It is worth noting that the first step in overcoming this problem began by asking "what's wrong with pixels?”, by Thomas Blaschke (Blaschke, Stroble 2001). $\mathrm{He}$ and his co-workers then proposed the concept of using image objects made by a homogenous group of neighboring pixels to add contextual information of high resolution remote sensing images in data processing.

Object-Based Image Analysis (OBIA) methods use the neighboring pixels to create image objects. Objects are usually made using different image segmentation methods. Image segmentation can be defined as the process of dividing an image into homogeneous regions. The result of blending two neighboring segments is that the new segment is not homogenous any more. Many different segmentation methods have been proposed for remote sensing imagery (Meinel, Neubert 2004). There are also several methods that have been especially developed for hyperspectral data (Mercier et al. 2003; Erturk, A., Erturk, S. 2006; Gorreta et al. 2009). These methods try to create objects that fit the terrestrial features and are recognizable by the naked eyes. After the completion of the object creation process, instead of single pixels, image objects are used as a basic processing unit for further image processing procedures (Benz et al. 2004).

Using the object-based concepts in classification has advantages in comparison to the classic pixelbased methods. The main benefit is a decrease in the computational cost in the classification process. This is because the classification process evaluates several pixels at once by assuming that the neighboring pixels belong to one land-cover class (Yu et al. 2006; Baraldi, Boschetti 2012). Also salt and pepper effect is omitted from the final result because the neighboring pixels are grouped into a single image object, and the local information is evaluated (Yu et al. 2006). In addition to these primary benefits, using OBIA improves the accuracy of classification. Using the topology of and the relation among image objects helps develop an object oriented image analysis (OOIA), which uses an OBIA procedure iteratively for a better understanding of image objects (Baraldi, Boschetti 2012).

Most remote sensing data processing methods can take advantage of object-based image analysis. Collecting reliable information in natural disasters (Gitas et al. 2004), producing and updating land cover maps (Whiteside, Ahmad 2005; Gholoobi et al. 2010; 
Gomes, Marçal 2003; Kruse 2005; Myint et al. 2011), and change detection (Bontemps et al. 2008; Walter 2004) are some of the application required, particularly in urban studies. Moreover, in recent years new features, such as ontology (Arvor et al. 2013) and agent recognition systems (Tabib Mahmoudi et al. 2013) have been added to the object-based image analysis, resulting in an improved capacity for identifying image objects.

Despite these improvements, not enough research has been done on the application of the OBIA in hyperspectral data analysis. Xie et al. uses the objectbased image analysis to classify objects created from hyperspectral and digital surface model data using a binary encoding method (Xie et al. 2009). Tarabalka et al. created the image objects by joining the pixels that belong to the same cluster in a local neighborhood. These pixels were labeled based on the majority voting in each image object using pixel-based SVM classification. Although, this method is similar to object-based methods, it is considered, and called, a spectral-spatial method (Tarabalka et al. 2009). Zhang and Huang used SVM to classify the image objects created using extracted features from hyperspectral data (Zhang, Huang 2010). Zhang and Xie also compared neural network pixel-based classification with objectbased texture features added to the neural network in vegetation classification; they conclude that object texture features can improve the vegetation mapping accuracy (Zhang, Xie 2012).

Due to the diversity of the sensor's spatial resolution and the size of the desired objects, the optimum method for various applications can be selected among sub-pixel, pixel-based or object-based methods. In addition, because of the variety of feature extraction and classification methods in hyperspectral data analysis, a comprehensive study on different ways to choose the

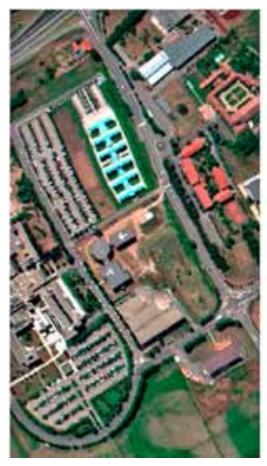

(a)

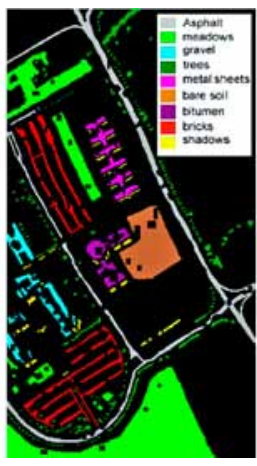

(b)

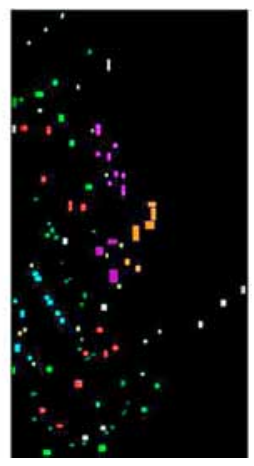

(c)
Fig. 1. (a) False color composite of ROSIS dataset. (b) Test sample. (c) Training sample classifier and feature extraction methods is conducted in this paper. To this end, two distinct frameworks for pixel-based and object-based classifiers are designed. The processing begins with feature extraction. The features are then classified using a pixel-based method. The number of features that yield the best accuracy are selected as an EFN and used in the object-based classification step. Image objects are created using the Fractal Net Evolution Algorithm (FNEA) (Baatz, Schäpe 2000) segmentation algorithm.

In this paper we intend to comprehensively compare object-based and pixel-based classifications of extracted features from hyperspectral data. In addition, the FNEA segmentation algorithm is used to create the image objects. We also herein evaluate the effect of different parameter values in these algorithms.

The rest of this paper is organized as follows. An overall view of methodology and the dataset used in this paper are introduced in the next section. Section 2 contains the theoretical bases of methods used. Section 3 presents the result of the experiments and lastly, in last section conclusions will be presented, preceded by a discussion of the results.

\section{Material and methods}

\subsection{Dataset}

The objective of this study was to evaluate the efficiency of object-based image analysis for hyperspectral data. For this purpose a benchmark dataset collected by Reflective Optics System Imaging Spectrometer (ROSIS) over the campus of University of Pavia, Italy was used. As depicted in Figure 1a, the data size was $610 \times 340$ pixels with spatial resolution of $1.3 \mathrm{~m}$ per pixel. The original data was gathered in 115 spectral bands ranging from 0.43 to $0.86 \mu \mathrm{m}$ in the electromagnetic spectrum. Twelve bands with low signal to noise ratio were omitted from the original dataset and the remaining bands (specifically, 103 of them) were used in the experiments. This dataset also contained ground truth data in 9 land cover classes. 42776 ground truth pixels were used as test samples (Fig. 1b) and 3921 pixels were used as train samples (Fig. 1c).

\subsection{Methodology}

An important issue and challenge in hyperspectral imagery is the problem of data redundancy. This problem is mainly due to the high correlation among the adjacent bands. The correlated bands extend the feature vector to a high dimensional space and make 
the estimation of statistical parameters difficult. This problem is called the Hughes phenomenon (Hughes 1968). The band reduction (or selection) and feature extractions are two possible solutions to overcome the curse of dimensionality problem (Landgrebe 2005). Both these methods have their own advantages and limitations. In this paper, we use the feature extraction methods that can be seen as a projection of the high dimensional data into a lower dimension feature space. Feature extraction provides data with a lower spectral dimension, and as a result, the subsequent classification requires less computation and less training samples.

In addition, a feature extraction can be conducted using two main strategies of supervised and unsupervised methods. In supervised methods, sufficient and high quality training data is needed to calculate the features with the best separation within the considered classes. In contrast, the unsupervised methods do not need a priori information about the land-cover classes. These methods create features by projecting the data into a new space with lower dimensionality such that most of the information content of the original data is preserved.

In this paper, the following well-known supervised feature extraction methods were used: the Discriminant Analysis Feature Extraction (DAFE), Decision Boundary Feature Extraction (DBFE), and Nonparametric Weighted Feature Extraction (NWFE). In addition, four unsupervised feature extraction methods were used: the Principal Component Analysis (PCA), Independent Component Analysis (ICA), Minimum Noise Fraction (MNF) and Spectral Band Mean (SBM).

Moreover, two classification categories of parametric and linear methods were used in the experiments. The parametric methods used were as follows: ML, FLL and SID. The linear methods used were SVM, SAM and BE. These methods were used for pixel and object-based approaches.

As depicted in Figure 2, the pixel-based approach begins with extracting features from the original dataset. Features are then used as input in the classification step. Because feature extraction methods do not normally obtain the optimum number of features, it is usually unclear as to how many features should be employed. In the experiments, we began using pixelbased classification with 3 features. Over time we added up to 20 features. As a result, regardless of which combination of feature and classifier we used, we obtained the number of features that yielded the best classification accuracy. This number of features can be interpreted as an EFN.

To perform the object-based classification as the workflow of Figure 3, the first step is creating the image objects. The optimum features calculated in the pixel-based step, are used as input data for the segmentation algorithm to create the image objects. The result was that the object-based approach outperformed the pixel-based one. Consequently, using the object-based

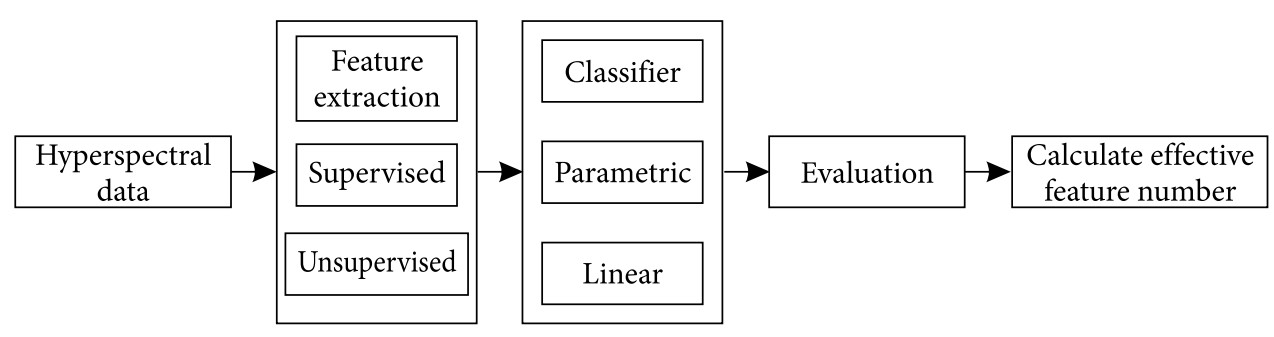

Fig. 2. Workflow of pixel-based classification framework

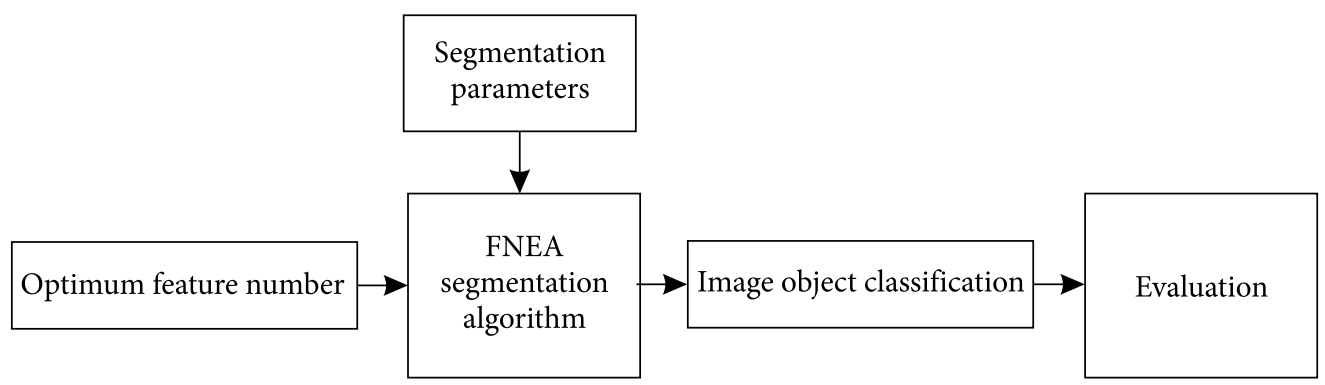

Fig. 3. Workflow of object-based classification framework 
methods improves the result of hyperspectral data classification. The FNEA segmentation algorithm is then used to create image objects. This algorithm uses the scale parameters and the weight of using shape information against the spectral information, beside the number of input features to segment the image. In order to avoid under-segmentation, an empirical method is used to find the best parameters. The mathematical detail of the algorithms will be presented in the next section.

After the image objects have been created, the feature vector of each object is then established as the mean feature vector of pixels forming the object. This framework leads to results that enable a comparison between the pixel and object-based approaches.

\section{Theoretical background}

This section contains a description of the methods and algorithms used in the experiments. Three main groups of algorithms were used here. These algorithms were the feature extraction, classification, and segmentation algorithms.

\subsection{Feature extraction}

Reducing the dimension of data is a critical step in hyperspectral data processing. It can prevent the Hughes phenomenon (Landgrebe 2005) and also improve the quality of further processing (Li 2004) (e.g., classification, target detection, and end member extraction). Two main approaches used in dimension reduction are band selection and feature extraction.

The feature or band selection approach try to find the subset of features that will lead to better results than the original features. Selection of the best features in the hyperspectral data has usually caused an NPhard problem. This is due to an excessive number of selections for $\mathrm{m}$ subset bands form $\mathrm{n}$ total bands. Feature extraction algorithms project data from the original spectral space into a new feature space using either linear or non-linear mapping of original features. In this paper, our objective is to evaluate and study the various feature extraction approaches.

Based on the either using a priori knowledge or not, two categories of feature extraction algorithms have been presented in the literature, called supervised and unsupervised. In unsupervised algorithms, the new feature space is defined such that the space axis orientation follows the scattered direction of data in original space. In this way, features are representative of most variations in the data.
The unsupervised feature extractions mainly take into account the inherent data scatter. In contrast, the supervised feature extraction methods take into account the desired land-cover classes in the dataset to extract features. Consequently, the features extracted by this approach discriminate well between the classes of interest.

Four unsupervised algorithms are used in this experiment, namely, PCA, ICA, MNF and SBM. PCA uses the principal components of data to produce uncorrelated features and segregate noise from data. The PCA moves the origin of feature space to the data mean and rotates the axes such that the variance is maximized (Richards 1999). The ICA transforms the data into new space, in which the components are statistically independent. This algorithm presumes the non-Gaussian distribution over the independent sources and the dataset (Hyvärinen, Oja 2000). The MNF aims to detect the intrinsic dimensionality of the data and omit the noises. This algorithm is a linear transformation comprised of two separate principal component analysis. First PCA is conducted on noisy data to create data with white noise. And final features are produced using PCA transformation with the noise-free features of the previous step (Green et al. 1988). The last unsupervised feature extraction method that had a simple set of computations was the concept is SBM. This method also used in (Tarabalka et al. 2009), tries to use the neighbor band correlation to extract the features. In this method, the mean value of some spectral features is used as a representative of the features.

In addition, three supervised feature extraction algorithms were used in the experiments: DAFE, DBFE and NWFE. DAFE uses within class and between classes scatter matrices to maximize the separability of subspace compared to the original space defined with all features. This procedure happens when the maximization of the trace ratio of these matrices takes place (Landgrebe 2005). DBFE aims to omit discriminantly redundant features while retaining discriminantly informative features. Redundant features are perpendicular to the normal of between class decision boundaries, whereas the informative features are parallel to the decision boundary, at least at one point on the boundary (Lee, Landgrebe 1993). The last supervised feature extraction method is the NWFE. This algorithm is an extension of the Nonparametric Discriminant Analysis (NDA) method, which proposes a non-parametric approach for resolving the problem of a parametric estimation of within class and between classes scatters 
matrices, similar to DAFE. In addition, the NWFE algorithm calculates the weight of contributing samples in inter and intra class matrices based on the distance between the sample and the mean of the class (Kuo, Landgrebe 2004).

\subsection{Land-cover classification}

Classification is the primary algorithm used to produce land-cover maps from remote sensing data. Unsupervised clustering and supervised classification algorithms are the main methods for this purpose. Unsupervised methods are able to separate the land-cover classes efficiently: however, they do not provide any information about the type of class. Supervised algorithms operate based on the machine learning theory. These algorithms need the training data and a training phase in advance. After these initial steps, they can separate and label any land-cover classes.

Six supervised algorithms were used in these experiments: three parametric and three linear ones. The parametric algorithms are ML, FLL, and SID. These algorithms use the estimated statistical parameters of the land-cover classes based on training samples. The trained classifier then enables us to classify any previously unknown dataset.

ML, a parametric algorithm, groups the pixels with the land-cover classes to which they probably belongs. The probability is calculated by presuming normal distribution for the classes (Richards 1999). The FLL algorithm acts similarly to ML; however, it assumes a multidimensional normal distribution of variance-covariance of the whole data to generate the decision boundaries to classify data. The purpose of this assumption is to generate the decision boundaries within which the data should be classified. This method does not use internal class variations in classification (Landgrebe 2005). The SID algorithm uses the divergence distance measure between the pixel and class mean vector to determine to which class the pixel belongs. This determination about the class of pixel is made based on the measured minimum distance (Du et al. 2004).

Linear algorithms use heuristic measures, which are characterized by their use of a pixel feature vector to classify the data. For example, the BE method converts the feature vector of pixels and class spectral representatives into a binary code, based on a predefined threshold. Consequently, the pixel belongs to the class whose code best matches the pixel code (Mazer et al. 1988). The SAM method uses angular measure of similarity for classification. In this method, the angular distance between the pixels feature vector and the reference feature vector of class is calculated in $\mathrm{n}-\mathrm{D}$ feature space. Using the angular measure, the effect of illumination, as well as albedo effects, can be minimized (Kruse et al. 1993). The SVM method maximizes the margin between classes by defining optimal hyperplanes. This method is a binary classifier; consequently, in multi-class problems several binary SVMs need to be integrated (Mercier, Lennon 2003).

\subsection{Image segmentation}

Image objects are defined as the homogeneous regions in an image that belongs to the same terrain features, or i.e., the same land-cover class. Image segmentation algorithms are used to create the image objects. the FNEA algorithm (Baatz, Schäpe 2000), a well-known segmentation algorithm used in the OBIA, was used in this experiment.

FNEA uses a region growing procedure in segmentation. The algorithm starts with some pixels as seed points all over the image plane as segment primitives and in a repetitive cycle, the neighboring objects are merged to create the bigger image objects. The heterogeneity measure, computed using the following equation, is used as a criterion to decide either two neighboring objects merge or not. This criterion calculates the heterogeneity change during the merging of two neighboring objects spectrally and spatially:

$$
h=w h_{\text {spectral }}+(1-w) h_{\text {spatial }},
$$

where parameter $w$ tunes the contribution weight of $h_{\text {spectral }}$ and $h_{\text {spatial }}$. The spectral component $h_{\text {spectral }}$ is calculated as follows:

$$
\begin{aligned}
h_{\text {spectral }}= & \sum_{b=1}^{B} W_{b}\left[N_{\text {merge }} \sigma_{\text {merge }}-\right. \\
& \left(N_{o b j 1} \sigma_{o b j 1}+N_{o b j 2} \sigma_{o b j 2}\right) .
\end{aligned}
$$

Here $B$ denotes the number of spectral bands and $W_{b}$ is the weight parameter of the bands. The $N$ values are the number of pixels in the merged object as well as neighboring objects. $\sigma$ is the standard deviation of the feature vector of pixels contained in the objects. The spatial component of the heterogeneity measure, $h_{\text {spatial }}$, is calculated according to the weighted sum of the smoothness and compactness criteria as follows:

$$
h_{\text {spatial }}=w_{\text {compact }} h_{\text {compact }}+\left(1-w_{\text {compact }}\right) h_{\text {smooth }}
$$

In this equation $w_{\text {compact }}$ defines the weight value of compactness heterogeneity measure, $h_{\text {compact }}$, against the smoothness, $h_{\text {smooth }}$. These measures are 
dependent on the compactness and smoothness of objects before and after merging. More details about calculating these measures can be found in the following equations:

$$
\begin{aligned}
h_{\text {smooth }}= & N_{\text {merge }} \frac{l_{\text {merge }}}{r_{\text {merge }}}-\left(N_{o b j 1} \frac{l_{o b j 1}}{r_{o b j 1}}+N_{o b j 2} \frac{l_{o b j 2}}{r_{o b j 2}}\right) ; \\
& h_{\text {compact }}=N_{\text {merge }} \frac{l_{\text {merge }}}{\sqrt{N_{\text {merge }}}}- \\
& \left(N_{\text {obj1 }} \frac{l_{\text {obj } 1}}{\sqrt{N_{\text {merge }}}}+N_{\text {obj2 } 2} \frac{l_{\text {obj } 2}}{\sqrt{N_{\text {merge }}}}\right) .
\end{aligned}
$$

New parameters used in these equations are 1 and $r$ both of which respectively, are perimeters of the both objects and the rectangle surrounding the image object.

In the segmentation cycle, we need to be able to calculate the heterogeneity measures in any iteration for any neighboring candidate objects that are to be merged. The decision to merge the objects is based on a comparison of this value with the scale parameter. The scale parameter is a criterion introduced into the algorithm by the user for the purpose of defining the maximum allowable change in heterogeneity while merging two objects. This criterion determines the size of the final objects, which is very important in the OBIA.
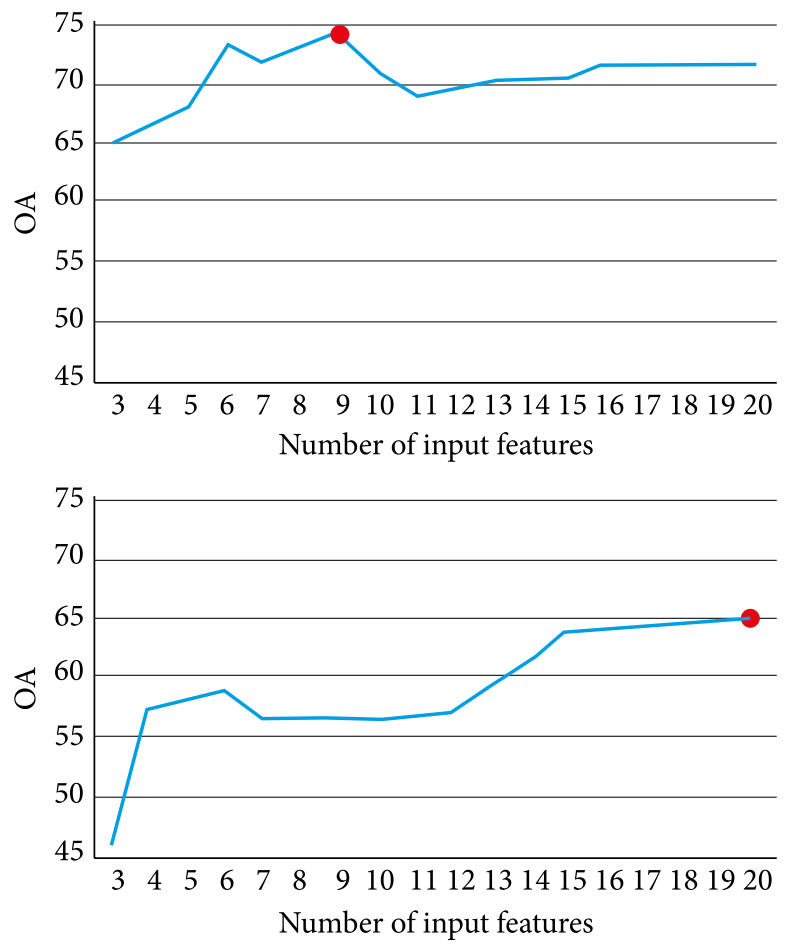

Fig. 4. Change in overall accuracy with increase in input feature number for PCA-FLL (a) and DBFE-SAM (b) feature extraction-classifier ensemble (effective feature number in each case is marked by circle)

\section{Experimental results}

The experiments were conducted on the ROSIS Pavia campus dataset. This dataset was collected by the ROSIS sensor over the campus area in the University of Pavia, northern Italy on July 2002. The dataset covers a range of 0.43 to $0.86 \mu \mathrm{m}$ on the EM spectrum, which consists of both the visible and the infrared EM spectrum. 103 spectral bands out of 115 spectral bands were selected for further processing, considering the Signal-to-Noise ratio and water absorption bands. Moreover, there are ground truth data in 9 different urban classes to train the classifier and evaluate the results of classification. The ground truth data are divided into 3921 train pixels and 42776 test samples, as depicted in Figure 1.

As already mentioned, this study intends to evaluate the performance of the pixel-based and objectbased classification of hyperspectral imagery. The overall accuracy and the Kappa coefficient described in (Congalton 1991) were used to evaluate the results and compare the different methodologies.

The proposed methodology starts with pixelbased classification. In this step, features that lead to the best overall accuracy qualified as the EFN. This is the case for any combination of the feature extractionclassifiers. EFN contains the features that successfully discriminate among the various land-cover classes. These features were used in the next step to create image objects and conduct the object-based classification process. The details of the results are presented in the following section.

\subsection{Pixel-based classification}

The feature vector of a pixel was used in this step to group a given individual pixel in a given land-cover class. Seven feature extraction methods in addition to six supervised classification algorithms were used under 42 different experimental conditions. Every experiment begins with three features as the input for classification. The features were then added one by one.

An important purpose of this step was to find the EFN in every experiment. The EFN was found by using the feature extraction-classifier. As depicted in Figure 4, these experiments reveal that when the number of input features increases, the $\mathrm{OA}$ changes in two ways. In the first case, when using the reasonable features number, the OA reached its maximum value. By adding more features, it began to decrease and undulate (Fig. 4a). In the second case, adding input features led to an increase in the OA value. As the 
OA increased, its rate was negligible compared to the computation load it adds. In such a case the process held 20 input features, which was an effective feature number (e.g., Fig. 4b).

Using a similar procedure, the EFN, the corresponding OA, and the Kappa coefficient, achieved were calculated for each experimental test and presented in Table 1 . The results of classifying the original features are also presented in this table. As can be seen, the SVM classifier yields the best accuracy when dealing with original features. This reveals the capacity of this classifier to successfully discriminate among the various land-cover classes using high dimensional data. In this case, the ML and FLL classifiers also provided acceptable results. This shows that statistical algorithms can work well when proper training data are available in advance.

The classification accuracy of other features show that in 30 out of 42 experimental tests, feature extraction can potentially either improve the accuracy of final results or yield the same result as the original features, but with using lower input features. Deteriorations are usually occur for the BE and SID classifiers. This is because of the adjacency of land-cover classes in feature space and the inability of these algorithms to discriminate among these classes.

\subsection{Object-based classification}

Image objects are the main processing unit in the OBIA; accordingly the first step in object-based image classification is creating image objects. As previously described, the FNEA segmentation algorithm is used to this end. The FNEA algorithm needs some parameters to be tuned prior to the run. These parameters are the scale parameter and the weight parameters, as discussed in the previous section. Different settings of these parameters change the shape and size of the created image objects. Setting the parameters of segmentation algorithms is an unresolved problem in performing image segmentation. This is mostly due to the lack of a standard criterion for measuring the adaptation of the segments to the terrain features (Zhang et al. 2008). For example, the evolutionary algorithms such as genetic algorithm (GA), are used in segmentation parameter optimization (Zhang et al. 2012). This methodology needs two criteria to assess the homogeneity of an image segment, one for segmentation and one to calculate the cost function. As none of these criteria are standard, different functions can be used and consequently different results may be obtained.
These problems led researchers to use a trial and error approach, in addition to visually inspecting the created image objects. In our experiments, we designed a mechanism for using a trial and error approach in order to create image objects that yield the best classification accuracy. To this end, the image segmentation was checked visually to prevent under-segmentation, and change the scale parameter, the weight of spectral versus spatial heterogeneity and the number of input features. In each case the parameterization that yields the best classification accuracy is stored as the result of an object-based classification.

The results of object-based classification are depicted in Table 2. Result of object-based classification experiments. It should be noted that both the OA and Kappa coefficient in the table correspond to the best parameterization of the FNEA algorithm and yielded the highest values during the experiments. Moreover, the number of input features was limited to the effective feature number, obtained in the previous step.

Table 1. Results of pixel-based classification experiments

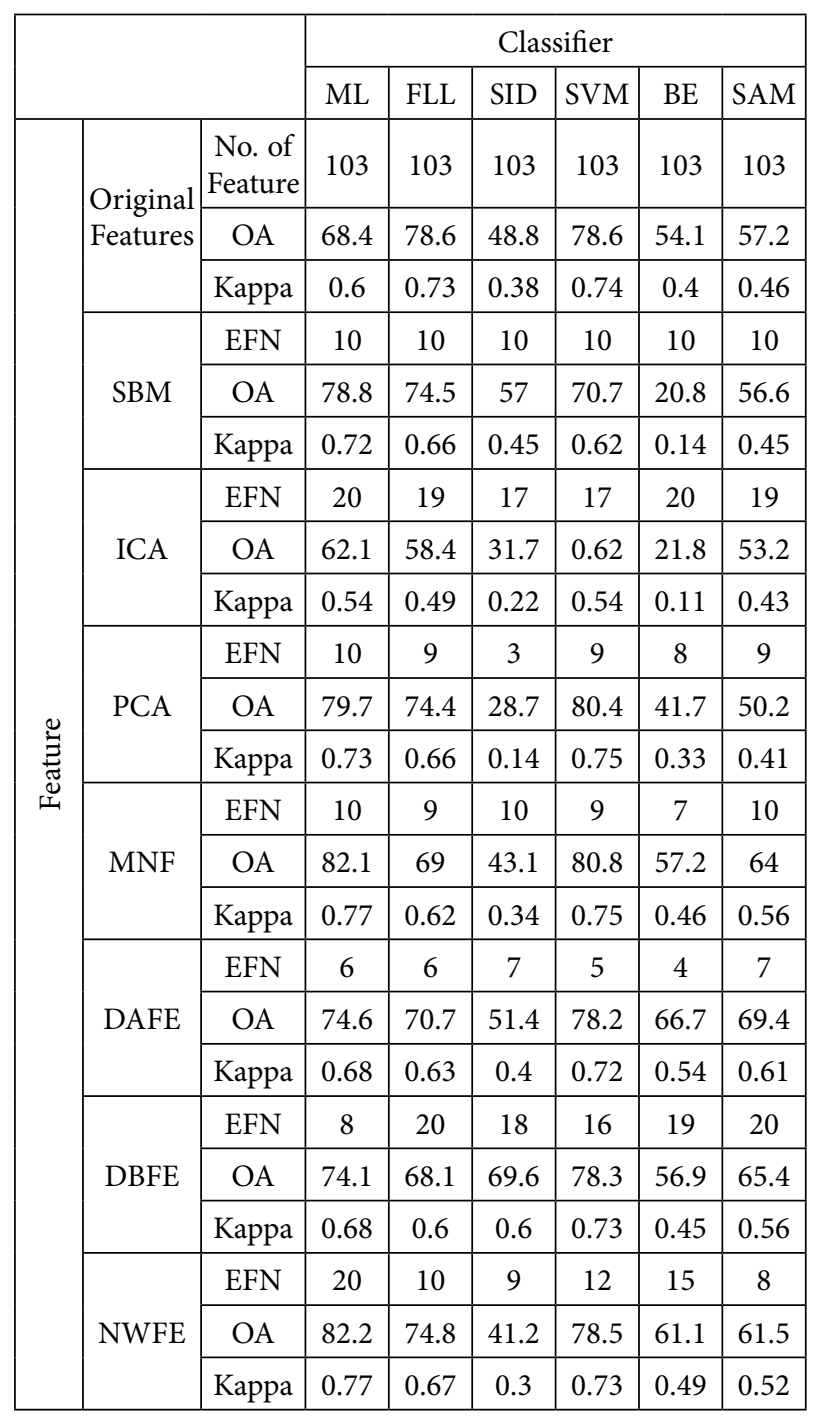


Table 2. Result of object-based classification experiments

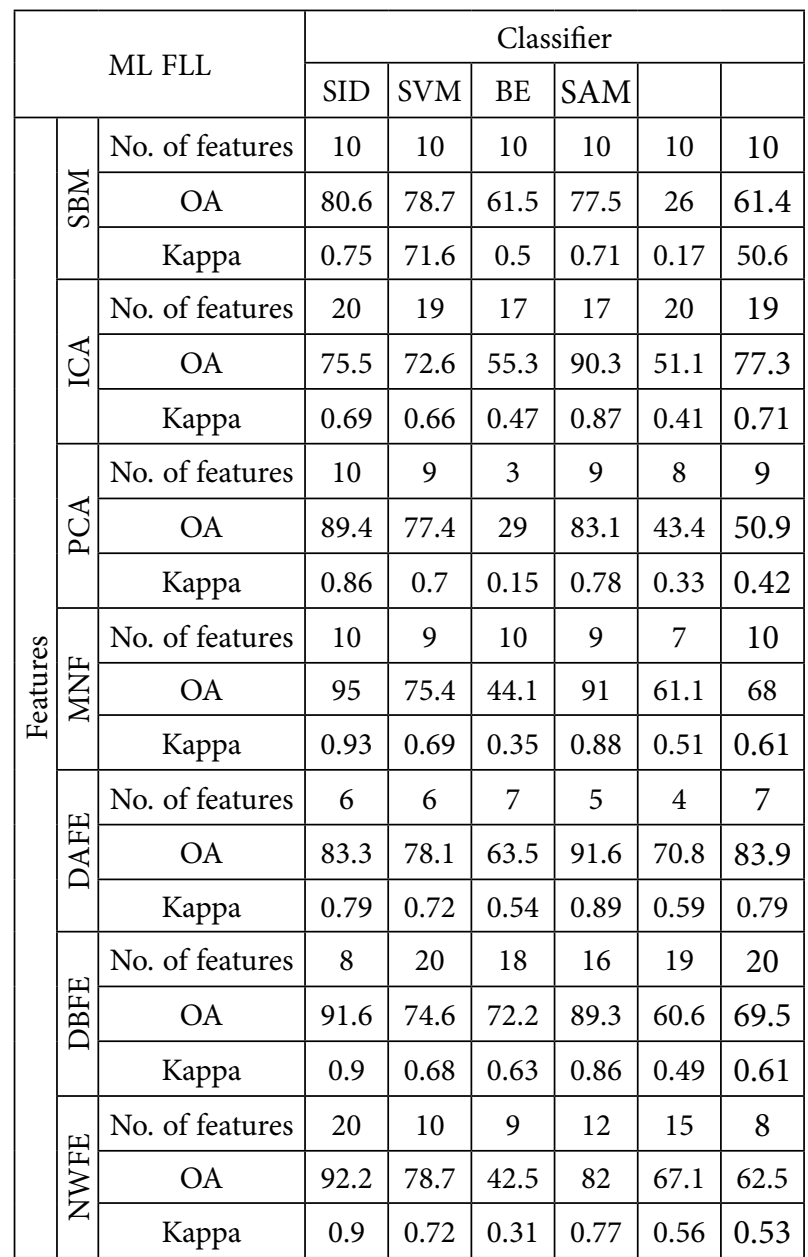

\subsection{Comparing results}

The performed experiments provide a framework for comparing the pixel-based and object-based classification results of six classification and seven feature extraction algorithms. Using diagrams depicted in Figure 5, a comprehensive comparison of the results is possible. The first point pertains to the overall performance of both the ML and SVM classifiers. These classifiers provide better results for each feature than other classifiers. Furthermore it is evident that in all cases, using an object-based paradigm improves the accuracy of classification. The difference is in the degree of improvement, which in some cases is significant (e.g. when using the ICA features and the SVM classifier), whereas in others it is negligible (e.g., when using PCA features and the SAM classifier). The occurrence of this phenomenon is related to the performance of feature extraction-classifier combinations. For example, SAM, BE, and SID classifiers use a reference feature vector as a training sample. The reference feature vector represents the average behavior of pixels belonging to the same land-cover class. Moreover, the signatures of image objects represent the mean values of all pixels within the image objects. Using the mean feature vector in this way may cause a change in landcover separability.
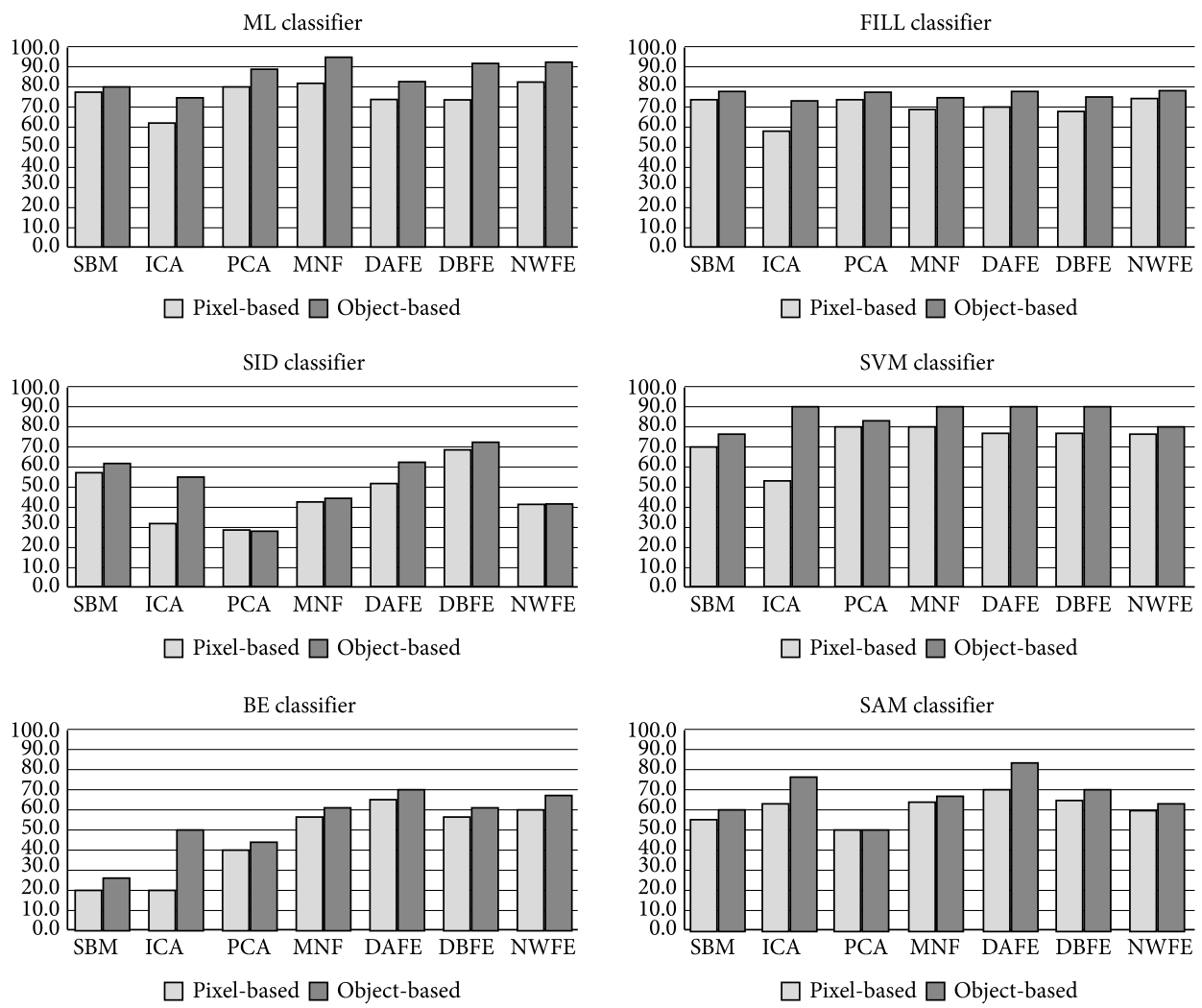

Fig. 5. The overall accuracies of pixel-based vs. object-based classifications 
In addition, for the purpose of comparing the performance of feature extraction algorithms and classifiers, the following results were calculated: the mean overall accuracy of any classifier using all feature extraction algorithms, and the mean overall accuracy of any feature extraction algorithm using all classifiers. The results are displayed in Table 3 and Table 4. It can be seen that the ML and SVM classifiers have the best performance with respect to both pixel-based and object-based paradigms. This demonstrates the strength of the ML classifier when there is proper training data and the SVM's capacity to discriminate among the land-cover classes. Aside from these two classifiers, FLL, SAM, BE, and SID performs best, respectively.

Among feature extraction algorithms, the DBFE and DAFE algorithms have the best average overall accuracy in both pixel-based and object-based methods. The performance of other methods differs from that of these two methods. When using the pixel-based NWFE, the MNF, SBM, PCA, and ICA respectively create better results. Also in object-based method MNF, NWFE, ICA, SBM and PCA had better performance respectively. This reveals a difference in capabilities regarding discriminating terrain objects when using the different feature extraction algorithms.

Lastly, the comparison of the pixel and objectbased results reveals that the object-based method can improve the overall accuracy of high resolution hyperspectral data classification by an average rate of about $8 \%$.

Table 3. Mean performance of feature extraction algorithms with all classifiers

\begin{tabular}{|c|c|c|c|}
\hline & & & Mean OA \\
\hline \multirow{14}{*}{$\begin{array}{c}\text { Feature } \\
\text { extraction } \\
\text { algorithms }\end{array}$} & \multirow{2}{*}{ SBM } & Pixel-based & 59.7 \\
\hline & & Object-based & 64.3 \\
\hline & \multirow{2}{*}{ ICA } & Pixel-based & 48.5 \\
\hline & & Object-based & 70.4 \\
\hline & \multirow{2}{*}{ PCA } & Pixel-based & 59.2 \\
\hline & & Object-based & 62.2 \\
\hline & \multirow{2}{*}{ MNF } & Pixel-based & 66 \\
\hline & & Object-based & 72.4 \\
\hline & \multirow{2}{*}{ DAFE } & Pixel-based & 68.5 \\
\hline & & Object-based & 78.5 \\
\hline & \multirow{2}{*}{ DBFE } & Pixel-based & 68.7 \\
\hline & & Object-based & 76.3 \\
\hline & \multirow{2}{*}{ NWFE } & Pixel-based & 66.6 \\
\hline & & Object-based & 70.8 \\
\hline
\end{tabular}

In addition to the superior quantitative analysis of object-based methods compared to pixel-based ones, the visual assessment of the final classification results also show some positive results yielded by the objectbased method. Figure 6 presents two examples of the

Table 4. Mean performance of classifiers with feature extraction algorithms

\begin{tabular}{|c|c|c|c|}
\hline & & & Mean OA \\
\hline \multirow{12}{*}{ Classifiers } & \multirow{2}{*}{ ML } & Pixel-based & 72.6 \\
\hline & & Object-based & 86.8 \\
\hline & \multirow{2}{*}{ FLL } & Pixel-based & 70 \\
\hline & & Object-based & 76.5 \\
\hline & \multirow{2}{*}{ SID } & Pixel-based & 46.1 \\
\hline & & Object-based & 52.6 \\
\hline & \multirow{2}{*}{ SVM } & Pixel-based & 74.5 \\
\hline & & Object-based & 86.4 \\
\hline & \multirow{2}{*}{$\mathrm{BE}$} & Pixel-based & 46.6 \\
\hline & & Object-based & 54.3 \\
\hline & \multirow{2}{*}{ SAM } & Pixel-based & 61.3 \\
\hline & & Object-based & 67.6 \\
\hline
\end{tabular}

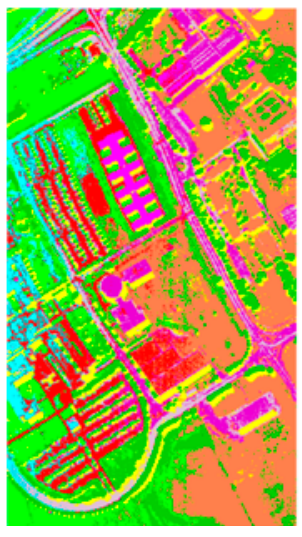

SAM classifier

Pixel-based

$\mathrm{OA}=64 \%$

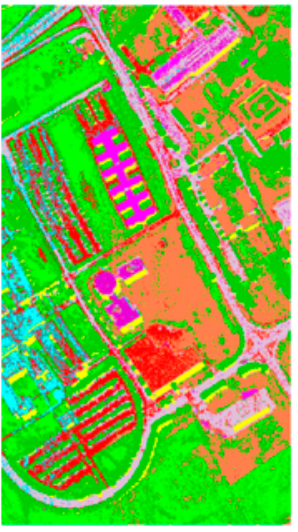

ML classifier

Pixel-based

$\mathrm{OA}=82.1 \%$

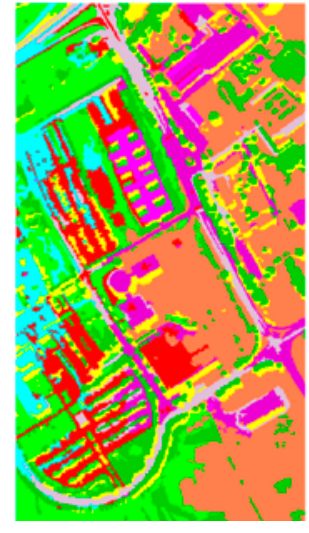

SAM classifier Object-based $\mathrm{OA}=68 \%$

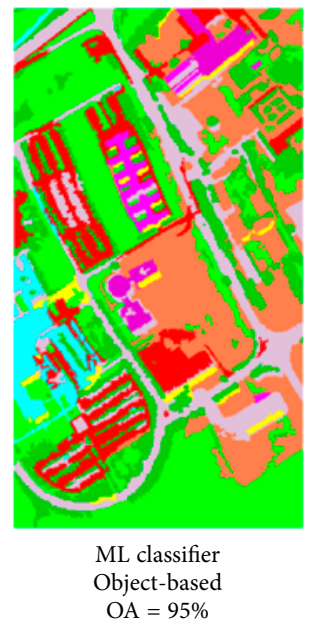

Fig. 6. Visual comparison of pixel and object-based classification of MNF features 
final classification results obtained by the MNF features using the ML and SAM classifiers. It is evident that using the OBIA can improve the visual quality of the final land-cover maps by omitting salt and pepper noises and reducing the post-processing analysis to produce the final map.

\section{Conclusions}

In this paper, we tried to demonstrate the efficacy of the OBIA in hyperspectral image analysis. To this end, we evaluated different classifiers and feature extraction algorithms that are commonly discussed in the literature. Experiments were conducted using both traditional pixel-based and the new object-based classification methods. A framework for comparing these two methods was designed.

The results revealed that the DAFE, DBFE, and MNF feature extraction algorithms attain better results, than other methods, even though the ML and SVM classifiers produced appropriate results in both cases. In addition, classification results for both the classifiers and the feature extraction algorithms show that object-based methods yielded more efficient results compared to pixel-based methods. The mean increase rate in the OA was approximately about 4.6, $21.9,3,6.4,10,7.6$, and 4.2 percent for the SBM, ICA, PCA, MNF, DAFE, DBFE, and NWFE features, respectively. Concerning the ML, FLL, SID, SVM, BE, and SAM classifiers, the increase rates was $14.2,6.5,6.5$, $11.9,7.7$, and 6.3 respectively. Notice that in all cases, there was an increase in the efficiency of the classification process. The difference in rates is largely due to the numerical calculations in classifiers and feature extraction algorithms, and the effect of using the mean feature value of image objects. It is worth noting that, in addition to providing numerical superiority, using the OBIA omits the salt and pepper noise from the final classification map and reduces the amount of postprocessing.

In conclusion, the superiority of the OBIA method in hyperspectral imagery reveals that the capabilities of these methods can be tested using other hyperspectral image analysis tasks e.g., target detection and end-member extraction. Furthermore, it seems that the final results of any application of the OBIA depend on the quality of the created image objects. Due to a lack of automatic segmentation algorithms, and their dependency on tuning parameters, it is possible to conduct fruitful research on this topic.

\section{Acknowledgements}

The authors would like to thank Prof. Gamba from the University of Pavia for making the Pavia hyperspectral image and reference dataset available. The authors would also like to thank the anonymous reviewers of a previous version of this paper for their insightful comments, each of which really helped us improve the paper.

\section{References}

Arvor, D.; Durieux, L.; Andrés, S.; Laporte, M.-A. 2013. Advances in Geographic Object-Based Image Analysis with ontologies: a review of main contributions and limitations from a remote sensing perspective, ISPRS Journal of Photogrammetry and Remote Sensing 82: 125-137.

http://dx.doi.org/10.1016/j.isprsjprs.2013.05.003

Baatz, M.; Schäpe, A. 2000. Multiresolution segmentation: an optimization approach for high quality multi-scale image segmentation, Angewandte geographische informationsverarbeitung 12(12): 12-23.

Baraldi, A.; Boschetti, L. 2012. Operational automatic remote sensing image understanding systems: beyond geographic object-based and object-oriented image analysis (GEOBIA/ GEOOIA). part 1: introduction, Remote Sensing 4(9): 26942735. http://dx.doi.org/10.3390/rs4092694

Benediktsson, J. A.; Palmason, J. A.; Sveinsson, R. 2005. Classification of hyperspectral data from urban areas based on extended morphological profiles, IEEE Transactions on Geoscience and Remote Sensing 43(3): 480-491.

http://dx.doi.org/10.1109/TGRS.2004.842478

Benz, U. C.; Hofmann, P.; Willhauck, G.; Lingenfelder, I.; Heynen, M. 2004. Multi-resolution, object-oriented fuzzy analysis of remote sensing data for GIS-ready information, ISPRS Journal of Photogrammetry and Remote Sensing 58(3): 239-258. http://dx.doi.org/10.1016/j.isprsjprs.2003.10.002

Bischof, H.; Schneider, W.; Pinz, A. J. 1992. Multispectral classification of Landsat-images using neural networks, IEEE Transactions on Geoscience and Remote Sensing 30(3): 482290. http://dx.doi.org/10.1109/36.142926

Blaschke, T. 2010. Object based image analysis for remote sensing, ISPRS Journal of Photogrammetry and Remote Sensing 65(1): 2-16. http://dx.doi.org/10.1016/j.isprsjprs.2009.06.004

Blaschke, T.; Stroble, J. 2001. What's wrong with pixels? Some recent developments interfacing remote sensing and GIS, GIS-Zeitschrift für Geoinformationssysteme 14(6): 12-17.

Bontemps, S.; Bogaert, P.; Titeux, N.; Defourny, P. 2008. An object-based change detection method accounting for temporal dependences in time series with medium to coarse spatial resolution, Remote Sensing of Environment 112(6): 3181-1391. http://dx.doi.org/10.1016/j.rse.2008.03.013

Camps-Valls, G.; Gomez-Chova, L.; Muñoz-Marí, J.; Vila-Francés, J. Calpe-Maravilla, J. 2006. Composite kernels for hyperspectral image classification, Geoscience and Remote Sensing Letters, IEEE 3(1): 93-97. http://dx.doi.org/10.1109/LGRS.2005.857031

Congalton, R. G. 1991. A review of assessing the accuracy of classifications of remotely sensed data, Remote Sensing of Environment 37(1): 35-46. http://dx.doi.org/10.1016/0034-4257(91)90048-B 
Dell'Acqua, F.; Gamba, P.; Ferrari, A.; Palmason, J.A.; Benediktsson, J. A.; Arnason, K. A. A. K. 2004. Exploiting spectral and spatial information in hyperspectral urban data with high resolution, Geoscience and Remote Sensing Letters, IEEE 1(4): 322-326. http://dx.doi.org/10.1109/LGRS.2004.837009

Du, Y.; Chang, C.-I.; Ren, H.; Chang, C.-C.; Jensen, J. O.; D'Amico, F. M. 2004. New hyperspectral discrimination measure for spectral characterization, Optical Engineering 43(8): 1777-1786. http://dx.doi.org/10.1117/1.1766301

Erturk, A.; Erturk, S. 2006. Unsupervised segmentation of hyperspectral images using modified phase correlation, Geoscience and Remote Sensing Letters, IEEE 3(4): 527-531. http://dx.doi.org/10.1109/LGRS.2006.880535

Gholoobi, M.; Tayyebib, A.; Taleyi, M.; Tayyebi, A. H. 2010. Comparing pixel based and object based approaches in land use classification in mountainous areas, in Proceeding of the ISPRS Commission VIII Mid-Term Symposium "Networking the World with Remote Sensing", 2010, Kyoto, Japan.

Gitas, I. Z.; Mitri, G. H.; Ventura, G. 2004. Object-based image classification for burned area mapping of Creus Cape, Spain, using NOAA-AVHRR imagery, Remote Sensing of Environment 92(3): 409-413.

http://dx.doi.org/10.1016/j.rse.2004.06.006

Gomes, A.; Marçal, A. 2003. Land cover revision through object based supervised classification of ASTER data, in Proc. ASPRS 2003 Annual Conference, 2003, Anchorage, USA.

Gorreta, N.; Roger, J. M.; Rabatel, G.; Ballon-Maurel, V.; Fiorio, C.; Lelong, C. 2009. Hyperspectral image segmentation: the butterfly approach, in Hyperspectral Image and Signal Processing: Evolution in Remote Sensing, 2009, Grenoble, France.

Green, A. A.; Berman, M.; Switzer, P.; Craig, M. D. 1988. A transformation for ordering multispectral data in terms of image quality with implications for noise removal, IEEE Transactions on Geoscience and Remote Sensing 26(1): 6574. http://dx.doi.org/10.1109/36.3001

Hughes, G. 1968. On the mean accuracy of statistical pattern recognizers, IEEE Transactions on Information Theory 14(1): 55-63. http://dx.doi.org/10.1109/TIT.1968.1054102

Hyvärinen, A.; Oja, E. 2000. Independent component analysis: algorithms and applications, Neural Networks 13(4): 411430. http://dx.doi.org/10.1016/S0893-6080(00)00026-5

Jackson, Q.; Landgrebe, D. A. 2002. Adaptive Bayesian contextual classification based on Markov random fields, IEEE Transactions on Geoscience and Remote Sensing 40(11): 2454-2463. http://dx.doi.org/10.1109/TGRS.2002.805087

Kettig, R. L.; Landgrebe, D. A. 1976. Classification of multispectral image data by extraction and classification of homogeneous objects, IEEE Transactions on Geoscience Electronics 14(1): 19-26. http://dx.doi.org/10.1109/TGE.1976.294460

Kruse, F. A. 2005. Multi-resolution segmentation for improved hyperspectral mapping, in The Defense and Security, Proc. SPIE 5806, Algorithms and Technologies for Multispectral, Hyperspectral, and Ultraspectral Imagery XI, 28 March 2005, Orlando, Florida, USA.

Kruse, F. A.; Lefkoff, A. B.; Boardman, J. W.; Heidebrecht, K. B.; Shapiro, A. T.; Barloon, P. J.; Goetz, A. F. H. 1993. The spectral image processing system (SIPS) - interactive visualization and analysis of imaging spectrometer data, Remote Sensing of Environment 44(2): 145-163. http://dx.doi.org/10.1016/0034-4257(93)90013-N
Kruse, F. A.; Boardman, J. W.; Huntington, J. F. 2003. Comparison of airborne hyperspectral data and EO-1 Hyperion for mineral mapping, IEEE Transactions on Geoscience and Remote Sensing 41(6): 1388-1400. http://dx.doi.org/10.1109/TGRS.2003.812908

Kuo, B.-C.; Landgrebe, D. A. 2004. Nonparametric weighted feature extraction for classification, IEEE Transactions on Geoscience and Remote Sensing 42(5): 1096-1105. http://dx.doi.org/10.1109/TGRS.2004.825578

Landgrebe, D. A. 2005. Signal theory methods in multispectral remote sensing. Wiley.

Lee, C.; Landgrebe, D. A. 1993. Feature extraction based on decision boundaries, IEEE Transactions on Pattern Analysis and Machine Intelligence 15(4): 388-400. http://dx.doi.org/10.1109/34.206958

Li, J. 2004. Wavelet-based feature extraction for improved endmember abundance estimation in linear unmixing of hyperspectral signals, IEEE Transactions on Geoscience and Remote Sensing 42(3): 644-649. http://dx.doi.org/10.1109/TGRS.2003.822750

Mazer, A. S.; Martin, M.; Lee, M.; Solomon, J. E. 1988. Image processing software for imaging spectrometry data analysis, Remote Sensing of Environment 24(1): 201-210. http://dx.doi.org/10.1016/0034-4257(88)90012-0

Meinel, G.; Neubert, M. 2004. A comparison of segmentation programs for high resolution remote sensing data, International Archives of Photogrammetry and Remote Sensing 35(Part B): 1097-1105.

Melgani, F.;Bruzzone, L. 2004. Classification of hyperspectral remote sensing images with support vector machines, IEEE Transactions on Geoscience and Remote Sensing 42(8): 1778-1790. http://dx.doi.org/10.1109/TGRS.2004.831865

Mercier, G.; Derrode, S.; Lennon, M. 2003. Hyperspectral image segmentation with Markov chain model, in Proceedings of Geoscience and Remote Sensing Symposium, IGARSS'03. 2003 IEEE International.

Mercier, G.; Lennon, M. 2003. Support vector machines for hyperspectral image classification with spectral-based kernels, in Proceedings of Geoscience and Remote Sensing Symposium, IGARSS'03. 2003 IEEE International.

Müller, R.; Cerra, D.; Reinartz, P. 2013. Synergetics framework for hyperspectral image classification, International Archives of the Photogrammetry, Remote Sensing and Spatial Information Sciences XL-1/W1, ISPRS Hannover Workshop 2013, 21-24 May 2013, Hannover, Germany, 257-262.

Myint, S. W.; Gober, P.; Brazel, A.; Grossman-Clarke, S.; Wenig, Q. 2011. Per-pixel vs. object-based classification of urban land cover extraction using high spatial resolution imagery, Remote Sensing of Environment 115 (5): 1145-1461. http://dx.doi.org/10.1016/j.rse.2010.12.017

Palmason, J. A.; Benediktsson, J. A.; Arnason, K. 2003. Morphological transformations and feature extraction of urban data with high spectral and spatial resolution, in Proceedings of Geoscience and Remote Sensing Symposium, IGARSS'03. 2003, IEEE International.

Paola, J. D.; Schowengerdt, R. A. 1995. A detailed comparison of backpropagation neural network and maximum-likelihood classifiers for urban land use classification, IEEE Transactions on Geoscience and Remote Sensing 33(4): 981-996. http://dx.doi.org/10.1109/36.406684

Pesaresi, M.; Benediktsson, J. A. 2001. A new approach for the morphological segmentation of high-resolution satellite 
imagery, IEEE Transactions on Geoscience and Remote Sensing 39(2): 309-320. http://dx.doi.org/10.1109/36.905239

Plaza, A. J. 2008. Parallel spatial-spectral processing of hyperspectral images, in Computational Intelligence for Remote Sensing. Springer, 163-192.

Richards, J. A. 1999. Remote sensing digital image analysis: an introduction. Springer.

http://dx.doi.org/10.1007/978-3-662-03978-6

Roessner, S.; Segl, K.; Heiden, U.; Kaufmann, H. 2001. Automated differentiation of urban surfaces based on airborne hyperspectral imagery, IEEE Transactions on Geoscience and Remote Sensing 39(7): 1525-1532.

http://dx.doi.org/10.1109/36.934082

Rogan, J.; Chen, D. 2004. Remote sensing technology for mapping and monitoring land-cover and land-use change, Progress in Planning 61(4): 301-325.

http://dx.doi.org/10.1016/S0305-9006(03)00066-7

Salehi, B.; Y Zhang, Y.; Zhong, M.; Dey, V. 2012. A review of the effectiveness of spatial information used in urban land cover classification of VHR imagery, International Journal of Geoinformatics 8(2).

Soille, P. 2003. Morphological image analysis: principles and applications. New York, Inc.: Springer-Verlag.

Tabib Mahmoudi, F.; Samadzadegan, F.; Reinartz, P. 2013. Object oriented image analysis based on multi-agent recognition system, Computers \& Geosciences 54: 219-230.

http://dx.doi.org/10.1016/j.cageo.2012.12.007

Tarabalka, Y. 2010. Classification of hyperspectral data using spectral-spatial approaches. Institut National Polytechnique de Grenoble-INPG.

Tarabalka, Y.; Benediktsson, J. A.; Chanussot, J. 2009. Spectral-spatial classification of hyperspectral imagery based on partitional clustering techniques, IEEE Transactions on Geoscience and Remote Sensing 47(8): 2973-2987. http://dx.doi.org/10.1109/TGRS.2009.2016214

Tarabalka, Y.; Benediktsson, J. A.; Chanussot, J.; Tilton, J. C. 2010. Multiple spectral-spatial classification approach for hyperspectral data, IEEE Transactions on Geoscience and Remote Sensing 48(11): 4122-4232. http://dx.doi.org/10.1109/igarss.2010.5649222

Tsai, F.; Chang, C.-K.; Liu, G.-R. 2006. Texture analysis for three dimensional remote sensing data by $3 D$ GLCM.

Walter, V. 2004. Object-based classification of remote sensing data for change detection, ISPRS Journal of Photogrammetry and Remote Sensing 58(3): 225-238.

http://dx.doi.org/10.1016/j.isprsjprs.2003.09.007

Whiteside, T.; Ahmad, W. 2005. A comparison of object-oriented and pixel-based classification methods for mapping land cover in northern Australia, in Proceedings of SSC2005 Spatial intelligence, innovation and praxis: The national biennial Conference of the Spatial Sciences Institute.

Xie, H.; Tong, X.; Heipke, C.; Lohmann, P.; Sorgel, U. 2009. Object-based binary encoding algorithm an integration of hyperspectral data and DSM, in Proc. of 2009 Joint Urban Remote Sensing Event, 20-22 May 2009, IEEE, 1-6. http://dx.doi.org/10.1109/URS.2009.5137551

Yu, Q.; Gong, P.; Clinton, N.; Biging, G.; Kelly, M.; Schirokauer, D. 2006. Object-based detailed vegetation classification with airborne high spatial resolution remote sensing imagery, Photogrammetric Engineering and Remote Sensing 72(7): 799. http://dx.doi.org/10.14358/PERS.72.7.799
Zhang, C.; Xie, Z. 2012. Combining object-based texture measures with a neural network for vegetation mapping in the Everglades from hyperspectral imagery, Remote Sensing of Environment 124: 310-320. http://dx.doi.org/10.1016/j.rse.2012.05.015

Zhang, H.; Fritts, J. E.; Goldman, S. A. 2008. Image segmentation evaluation: a survey of unsupervised methods, Computer Vision and Image Understanding 110(2): 260-280. http://dx.doi.org/10.1016/j.cviu.2007.08.003

Zhang, L.; Huang, X. 2010. Object-oriented subspace analysis for airborne hyperspectral remote sensing imagery, Neurocomputing 73(4): 927-936. http://dx.doi.org/10.1016/j.neucom.2009.09.011

Zhang, X.; Tong, H.; Chen, X. 2012. Multi-scale segmentation algorithm parameters optimization based on evolutionary computation, in Computational Intelligence and Intelligent Systems. Springer, 347-358.

Ahmad HADAVAND received the B.S. degree in surveying and geomatics engineering from Iran University of Science and Technology in 2009, and MSc degree in Photogrammetry from K. N. Toosi University of Technology in 2012. Currently he is working toward $\mathrm{PhD}$ degree and his main research interest includes object based image analysis, automatic image segmentation and digital photogrammetry.

Mehdi MOKHTARZADE received the MSc and $\mathrm{PhD}$ degrees in photogrammetry from the K. N. Toosi University of Technology, Tehran, Iran, in 2004 and 2008, respectively. He is currently an Associate Professor with the K. N. Toosi University of Technology, Tehran. His current research interests include georectification mathematical models, automatic feature extraction, hyperspectral remote sensing, digital photogrammetry, and computational intelligence.

Mohammad Javad Valadan ZOEJ received the $\mathrm{PhD}$ degree in remote sensing and photogrammetry from

the University of Glasgow, Glasgow, U.K. He is currently a Professor with the Faculty of Geodesy and Geomatic, K. N. Toosi University of Technology, Tehran, Iran. His research interests include 3-D spatial information extraction from space images, hyper spectral and multispectral image processing, automatic feature extraction, radargrammetry and interferometry.

Saeid HOMAYOUNI received the B.S. degree in surveying and geomatics engineering from the University of Isfahan, Isfahan, Iran, in 1996, the MSc degree in remote sensing and geographic information systems from Tarbiat Modaress University, Tehran, Iran, in 1999, and the PhD degree in signal and image from Telecom of Paris, Paris, France, in 2006. He has worked as a post-doc fellow in Signal and Image (LAPS) Laboratory of University of Bordeaux, France, from 2006 to 2007. From 2008 to 2011, he has been as an Assistant Professor with the Department of Geomatics and Surveying, College of Engineering, University of Tehran, Iran, and then he has worked as a NSERC Research Fellow with Science and Technology Branch of Agriculture Canada, in Ottawa. Since 2013, he has been as an Assistant Professor in RS and GIS with the Department of Geography of University of Ottawa, Ottawa, ON, Canada. His research activities are mainly focused on optical and SAR remote sensing for urban and agro-environmental applications. 
Mohammad SAADATSERESHT received the B.S. degree in surveying and geomatics engineering in 1996, the MSc degree in photogrammetry in 1998, and the $\mathrm{PhD}$ degree in close range photogrammetry in 2004, all from the University of Tehran, Tehran, Iran. He has worked as an Assistant Professor in the Institute of Photogrammetry, Department of Geomatics Engineering, College of Engineering, University of Tehran, since 2004. He has five patents in 3-D measurement and reconstruction subject, such as 3-D linear laser scanner and digital caliper. His research activities are mainly focused on close range photogrammetry and computer vision, UAV mapping, and aerial and satellite remote sensing and he has published more than 150 articles in these topics. 